Federico Villa Giuseppe Citerio

\section{Surpassing boundaries: volatile sedation in the NeuroICU}

Received: 4 September 2012

Accepted: 4 September 2012

Published online: 28 September 2012

(C) Copyright jointly held by Springer and ESICM 2012

This editorial refers to the article available at: doi:10.1007/s00134-012-2708-8.

F. Villa · G. Citerio $(\bullet)$

Neuroanaesthesia and Neurointensive Care Unit, Anestesia e Rianimazione, San Gerardo Hospital, via Pergolesi 33, 20900 Monza, Milano, Italy e-mail: g.citerio@hsgerardo.org

Recent evidence suggests that sedative drug usage in general intensive care unit (ICU) patients should be minimized. Patients should be mobilized early, and each patient's sedation and analgesia requirements should be evaluated on a daily basis to tailor the care of each individual patient $[1,2]$. However, this paradigm change does not apply to acute brain injury patients because in this cohort sedation, along with general targets, has additional aims. Sedative agents, in fact, are administered to critically ill adults with acute brain damage to induce anxiolysis, prevent agitation, and facilitate mechanical ventilation [3]. Additionally, sedation has some neuroprotective properties, improving intracranial pressure (ICP) and cerebral perfusion pressure (CPP), mainly by reducing the cerebral metabolic rate and, in turn, cerebral blood flow and volume [4].

Nevertheless, no clear data on the best sedative choice for acute brain damaged patients are available. A recent systematic review showed no convincing evidence that one sedative agent is more efficacious than another for outcomes, ICP, or CPP improvement in critically ill adults with severe traumatic brain injury [5]. On the other hand, sedative agents may cause adverse drug events, obscure the neurologic examination, and prolong the length of mechanical ventilation or ICU stay.

This double-edged sword hangs over our decisions and over patients with acute brain injury. Therefore, the saga of searching for an effective sedative agent with neuroprotective activities continues.

In recent years, a large number of animal studies investigated the potential neuroprotective effect of volatile sedative agents [6-15]. In vivo, it has been demonstrated that preconditioning with isoflurane improves long-term neurologic outcome after hypoxic-ischemic bran injury in neonatal rats, in adult mice, and after focal brain ischemia in adult rats. In vitro, isoflurane postconditioning provided neuroprotection in rat corticostriatal slices, while preconditioning induced protection against glutamate neurotoxicity in rat cerebellar slices; the combination of both pre- and postconditioning induced neuroprotection in a model using rat hippocampal neurons.

Although still under investigation, current evidence suggests that the mechanism of the induction of tolerance to ischemia is inducible nitric oxide synthase dependent; other factors that could be involved are the inhibition of excitatory neurotransmission and regulation of intracellular calcium responses during ischemia.

These seminal animal research studies, even if attractive for their potential benefit for ischemic damage, have not been translated into clinical studies, mainly because the use of a volatile agent carries the potential risk of vasodilatation causing an increase in cerebral blood flow $(\mathrm{CBF})$ and consequently an ICP rise. For this reason, it has been historically considered unsafe in acute brain damaged patients and confined outside neuroICUs.

Until recently, surpassing this boundary seemed foolhardy. Continuous monitoring of ICP and CBF and the demarcation of safety thresholds have allowed securely setting sailing for this adventure. In this ICM issue, Bösel 
and colleagues [16] presented the results of their investigation on the effects of volatile isoflurane sedation on cerebral oxygenation, circulation and pressure in a population of cerebrovascular patients. They enrolled ischemic stroke, intracerebral and subarachnoid hemorrhage patients, sedated initially with an intravenous sedative agent (propofol or midazolam) and switched to inhalative isoflurane at a minimal alveolar concentration (MAC) of 0.5 for 3 days. They observed a clinically irrelevant increase in ICP; also, cerebral artery flow velocity did not change significantly. This result is interesting and is consistent with the results of other studies; a possible explanation could be that isoflurane causes a reduction in vascular resistance in the microcirculation and in the intraparenchymal arterioles rather than large vessels as the mean cerebral artery that is explored by transcranial Doppler [17].

Mean arterial pressure (MAP) and CPP were decreased, particularly in patients who were previously on midazolam. They concluded that it is possible to reach satisfactory sedation by applying volatile isoflurane for the long-term without causing a relevant raise in ICP if baseline ICP values are low or only moderately elevated and if arterial blood pressure and $\mathrm{PaCO}_{2}$ are kept stable.

The potential ICP increase could derive from different causes: an increase in CBF, given that isoflurane itself may cause uncoupling of CBF from the cerebral metabolism, or an increase in arterial $\mathrm{PCO}_{2}$ due to the dead-space expansion caused by the insertion of the $\mathrm{AnaConDa}^{\circledR}$ system in the ventilator circuit. For all these reasons, an increase in ICP should be considered and monitored when applying this sedative agent, and we agree with the author's recommendation that multimodal neuromonitoring is mandatory when applying volatile sedation.

These data need to be confirmed in larger prospective studies; however, the results are consistent with our recently published experience. In a group of patients affected by subarachnoid hemorrhage, switching to isoflurane sedation did not significantly change ICP and cerebral artery flow velocity, but it did increase regional CBF.

When our ancestors came in contact with the fire for the first time, they were terrified by the possibility of burning and kept away. Later, applying some precautions, they realized the usefulness of fire to keep them warm and to cook food; they were able to reap the benefits, limiting the risks. We are in the same situation. As suggested in the animal studies, the potential neuroprotective benefit of inhalation sedation is huge and unexplored. We have to find ways to exploit it, while, driven by monitoring, avoiding the side effects.

The journey is still long, but these experiences have opened new, hitherto unexplored scenarios. As Albert Einstein said "A theory is something nobody believes, except the person who made it. An experiment is something everybody believes, except the person who made it." Now we have a theory (and we are believers), and we are looking for stronger experimental evidence.

\section{References}

1. Strøm T, Toft P (2011) Time to wake up the patients in the ICU: a crazy idea or common sense? Minerva Anestesiol 77:59-63

2. Morandi A, Brummel NE, Ely EW (2011) Sedation, delirium and mechanical ventilation: the "ABCDE" approach. Curr Opin Crit Care 17:43-49

3. Mirski MA, Lewin JJ (2010) Sedation and analgesia in acute neurologic disease. Curr Opin Crit Care 16:81-91

4. Citerio G, Cormio M (2003) Sedation in neurointensive care: advances in understanding and practice. Curr Opin Crit Care 9:120-126

5. Roberts DJ, Hall RI, Kramer AH, Robertson HL, Gallagher CN, Zygun DA (2011) Sedation for critically ill adults with severe traumatic brain injury: a systematic review of randomized controlled trials. Crit Care Med 39:2743-2751
6. Zhao P, Peng L, Li L, Xu X, Zuo Z (2007) Isoflurane preconditioning improves long-term neurologic outcome after hypoxic-ischemic brain injury in neonatal rats. Anesthesiology 107:963-970

7. Zhao P, Zuo Z (2004) Isoflurane preconditioning induces neuroprotection that is inducible nitric oxide synthase-dependent in neonatal rats. Anesthesiology 101:695-703

8. McAuliffe JJ, Joseph B, Vorhees CV (2007) Isoflurane-delayed preconditioning reduces immediate mortality and improves striatal function in adult mice after neonatal hypoxiaischemia. Anesth Analg 104:1066-1077

9. Zheng S, Zuo Z (2004) Isoflurane preconditioning induces neuroprotection against ischemia via activation of P38 mitogen-activated protein kinases. Mol Pharmacol 65:1172-1180
10. Kapinya KJ, Löwl D, Fütterer C, Maurer M, Waschke KF, Isaev NK, Dirnagl U (2002) Tolerance against ischemic neuronal injury can be induced by volatile anesthetics and is inducible NO synthase dependent. Stroke 33:1889-1898

11. Chi OZ, Hunter C, Liu X, Weiss HR (2010) The effects of isoflurane pretreatment on cerebral blood flow, capillary permeability, and oxygen consumption in focal cerebral ischemia in rats. Anesth Analg 110:1412-1418

12. Lee JJ, Li L, Jung HH, Zuo Z (2008) Postconditioning with isoflurane reduced ischemia-induced brain injury in rats. Anesthesiology 108:1055-1062

13. Zheng S, Zuo Z (2005) Isoflurane preconditioning decreases glutamate receptor overactivation induced purkinje neuronal injury in rat cerebellar slices. Brain Res 1054:143-151

14. McMurtrey RJ, Zuo Z (2010) Isoflurane preconditioning and postconditioning in rat hippocampal neurons. Brain Res 1358:184-190 
15. Fugate JE, Burns JD, Wijdicks EF, Warner DO, Jankowski CJ, Rabinstein AA (2010) Prolonged high-dose isoflurane for refractory status epilepticus: is it safe? Anesth Analg 111:1520-1524
16. Böesel J, Purrucker JC, Nowak F,

Renzland J, Schiller P, Beneviste Perez E, Poli S, Brunn B, Hacke W, Steiner T (2012) Volatile isoflurane sedation in cerebrovascular intensive care patients using AnaConDa ${ }^{\circledR}$ : effects on cerebral oxygenation, circulation, and pressure. Intensive Care Med
17. Villa F, Iacca C, Molinari AF, Giussani C, Aletti G, Pesenti A, Citerio G (2012) Inhalation versus endovenous sedation in subarachnoid hemorrhage patients: effects on regional cerebral blood flow. Crit Care Med 40(10):2797-2804 